\title{
Con-S-K-Invariant Partial Orderings on Matrices
}

\author{
Dr G.Ramesh*, Dr B.K.N.Muthugobal** \\ *Associate Professor, Ramanujan Research Centre, Department of Mathematics, Govt. Arts College \\ (Autonomous), Kumbakonam-612 001, \\ **4, Noor Nagar,Kumbakonam- 612 001, India.
}

Abstract: In this paper it is shown that all standard partial orderings are preserved for con-s-k-EP matrices. Keywords: Con-s-k-EP Matrix, Partial Ordering.

\section{Introduction}

Let $c_{n x n}$ be the space of nxn complex matrices of order n. let $C_{n}$ be the space of all complex $\mathrm{n}$ tuples. For $\mathrm{A} \epsilon c_{n x n}$. Let $\bar{A}, A^{T}, A^{*}, A^{S}, \bar{A}^{S}, A^{\dagger}, \mathrm{R}(\mathrm{A}), \mathrm{N}(\mathrm{A})$ and $\rho(A)$ denote the conjugate, transpose, conjugate transpose, secondary transpose, conjugate secondary transpose, Moore Penrose inverse range space, null space and rank of $\mathrm{A}$ respectively. A solution $\mathrm{X}$ of the equation $\mathrm{AXA}=\mathrm{A}$ is called generalized inverse of $\mathrm{A}$ and is denoted by $A^{-}$. If $\mathrm{A} \in c_{n x n}$ then the unique solution of the equations $\mathrm{A} X \mathrm{XA}$ $=\mathrm{A}, \mathrm{XAX}=\mathrm{X},[A X]^{*}=\mathrm{AX},(\mathrm{XA})^{*}=\mathrm{XA}[9]$ is called the Moore-Penrose inverse of $\mathrm{A}$ and is denoted by $A^{\dagger}$. A matrix A is called Con-s- $k-E P_{r}$ if $\rho(\mathrm{A})=\mathrm{r}$ and $\quad \mathrm{N}(\mathrm{A})=\mathrm{N}\left(A^{T} \mathrm{VK}\right.$ ) (or) $\mathrm{R}(\mathrm{A})=\mathrm{R}\left(\mathrm{KV} A^{T}\right)$. Throughout this paper let " $k$ " be the fixed product of disjoint transposition in $S_{n}=\{$ $1,2, \ldots . \mathrm{n}\}$ and $\mathrm{k}$ be the associated permutation matrix .

Let us define the function $\boldsymbol{k}(\mathrm{x})=\left(x_{k(1)}, x_{k(2)}, \ldots, x_{k(n)}\right)$. A matrix $\mathrm{A}=\left(a_{i j}\right) \epsilon c_{n x n}$ is s-k-symmetric if $a_{i j}=a_{n-k(j)+1, n-k(i)+1}$ for $\mathrm{i}, \mathrm{j}=1,2, \ldots \ldots \mathrm{n}$. A matrix $\mathrm{A} \in c_{n x n}$ is said to be Con-s-k-EP if it satisfies the condition $A_{x}=0<=>A^{s} k(x)=0$ or equivalently N(A) $=\mathrm{N}\left(A^{T} \mathrm{VK}\right)$. In addition to that $\mathrm{A}$ is con-s-k-EP $<=>K V A$ is con-EP or AVK is con-EP and $\mathrm{A}$ is con-s-k-EP $<=>A^{T}$ is con-s-k-EP $\mathrm{P}_{\mathrm{r}}$ moreover $\mathrm{A}$ is said to be Con-s-k-EP $\mathrm{P}_{\mathrm{r}}$ if $\mathrm{A}$ is con-s-k-EP and of rank r. For further properties of con-s-k-EP matrices one may refer [6].

Theorem 2 [2]

Let $A, B \in C_{n x n}$. Then we have the following:

(i) $R(A B) \subseteq R(A) ; N(B) \subseteq N(A B)$.

(ii) $R(A B)=R(A) \Leftrightarrow \rho(A B)=\rho(A)$ and

$$
N(A B)=N(B) \Leftrightarrow \rho(A B)=\rho(B)
$$

Theorem 2.1 [p.21, 8]

$$
\text { (iii) } N(A)=N\left(A^{*} A\right) \quad \text { and } R(A)=R\left(A^{*} A\right)
$$

Let $A, B \in C_{n x n}$. Then

(i) $N(A) \subseteq N(B) \Leftrightarrow R\left(B^{*}\right) \subseteq R\left(A^{*}\right)$

$$
\Leftrightarrow B=B A^{-} A \text { for all } A^{-} \in A\{1\}
$$

(ii) $N\left(A^{*}\right) \subseteq N\left(B^{*}\right) \Leftrightarrow R(B) \subseteq R(A) \Leftrightarrow B=A A^{-} B$ for every $A^{-} \in A\{1\}$.

\section{Definition 2.1.1}

$$
\text { For } A, B \in C_{n \times n} \text {, }
$$

(i) $A \geq_{L} B$ if $A-B \geq 0$.

(ii) $A \geq_{T} B$ if $B^{T} B=B^{T} A$ and $B^{T} B=A B^{T}$

(iii) $A \geq_{r s} B$ if $\rho(A-B)=\rho(A)-\rho(B)$. 
The relationship between the transpose and minus orderings is studied by Baksalary [1], Mitra [8], Mitra and Puri [7] and Hartwig and Styan [4, 5].

In the sequel, the following known results will be used.

Result 2.1.2 [5]

For $A, B \in C_{n \times n}, A \geq_{L} B \Leftrightarrow \rho\left(A^{\dagger} B\right) \leq 1$ and $R(B) \subseteq R(A)$ where $\mathbf{r}(A)=\max \{|\lambda|: \lambda$ is an eigen value of $A\}$ is the spectral radius.

Result 2.1.3 [3]

For $A, B \in C_{n \times n}, A \geq_{T} B \Leftrightarrow A \geq_{r s} B$ and $(A-B)^{\dagger}=A^{\dagger}-B^{\dagger}$. For other conditions to be added to rank subtractivity to be equivalent to star order, one may refer [1].

Result 2.1.4 [4]

$$
\text { For } A, B \in C_{n \times n}, \quad A \geq_{r s} B \Leftrightarrow B=B A^{-} B=B A^{-} A=A A^{-} B .
$$

\section{Definition 2.1.5}

Let $A \in C_{n \times n}$, if $A A^{S}=A^{S} A=I$ then $A$ is called s-orthogonal matrix.

Theorem 2.1.6

For $A, B \in C_{n \times n}, K$ is the permutation matrix associated with 'k' the set of all permutations in $S=\{1,2, \ldots, n\}$ and $V$ is the secondary diagonal matrix with units in its secondary diagonal then,

(i) $A \geq_{L} B \Leftrightarrow K V A \geq_{L} K V B \Leftrightarrow A V K \geq_{L} B V K$.

(ii) $A \geq_{T} B \Leftrightarrow K V A \geq_{T} K V B \Leftrightarrow A V K \geq_{T} B V K$.

(iii) $A \geq_{r s} B \Leftrightarrow K V A \geq_{r s} K V B \Leftrightarrow A V K \geq_{r s} B V K$.

Proof

(i) $A \geq_{L} B \Leftrightarrow \mathbf{r}\left(A^{\dagger} B\right) \leq 1$ and $R(B) \subseteq R(A)$

(by result (2.1.2))

$\Leftrightarrow \mathbf{r}\left(A^{\dagger} V K K V B\right) \leq 1$ and $B=A A^{\dagger} B$

(by Theorem

(2.1)) $\Leftrightarrow \mathbf{r}\left(A^{\dagger} V K K V B\right) \leq 1$ and $(K V B)=(K V A)\left(A^{\dagger} V K\right)(K V B)$

$\Leftrightarrow \mathbf{r}\left((K V A)^{\dagger}(K V B)\right) \leq 1$ and $R(K V B) \subseteq R(K V A)$

$\Leftrightarrow K V A \geq_{L} K V B$

(by (2.11) [6] and Theorem (2.1))

(by result (2.1.2))

Also, $A \geq_{L} B \Leftrightarrow \mathbf{r}\left(A^{\dagger} B\right) \leq 1$ and $R(B) \subseteq R(A)$

(by result (2.1.2))

$\Leftrightarrow \mathbf{r}\left(K V A^{\dagger} B V K\right) \leq 1$ and $B=A A^{\dagger} B$

(by Theorem(2.1))

$\Leftrightarrow \mathbf{r}$

$\left((A V K)^{\dagger}(B V K)\right) \leq 1$ and

$(B V K)=(A V K)(A V K)^{\dagger}(B V K)$

$\Leftrightarrow \mathbf{r}\left((A V K)^{\dagger}(B V K)\right) \leq 1$ and $R(B V K) \subseteq R(A V K)$

(by Theorem (2.1))

$\Leftrightarrow A V K \geq_{L} B V K$

(by Result (2.1.2))

(ii) $A \geq_{T} B \Leftrightarrow B^{T} B=B^{T} A$ and $B B^{T}=A B^{T} \quad$ (by definition of transpose ordering) $\Leftrightarrow B^{T} V K K V B=B^{T} V K K V A$ and $K V B B^{T} V K=K V A B^{T} V K$

$\Leftrightarrow(K V B)^{T}(K V B)=(K V B)^{T}(K V A)$ and $(K V B)(K V B)^{T}=(K V A)(K V B)^{T}$

$$
\Leftrightarrow(K V A) \geq_{T} K V B \quad \text { (by definition of transpose }
$$

ordering) Similarly it can be proved that, $A \geq_{T} B \Leftrightarrow A V K \geq_{T} B V K$. 
(iii) $A \geq_{r s} B \Leftrightarrow \rho(A-B)=\rho(A)-\rho(B) \quad$ (by definition of minus ordering)

$$
\begin{gathered}
\Leftrightarrow \rho(K V(A-B))=\rho(K V A)-\rho(K V B) \\
\Leftrightarrow \rho(K V A-K V B)=\rho(K V A)-\rho(K V B) \\
\Leftrightarrow K V A \geq_{r s} K V B .
\end{gathered}
$$

Similarly it can be proved that, $A \geq_{r s} B \Leftrightarrow A V K \geq_{r s} B V K$.

Thus, all the three standard partial orderings are preserved for con-s-k-EP matrices.

Result 2.1.7

The following results can be easily verified by using the Theorem (2).

Lowener ordering is preserved under unitary similarity, that is, $A \geq_{L} B \Leftrightarrow P^{T} A P \geq_{L} P^{T} B P$.

\section{Result 2.1.8}

Star ordering is preserved under unitary similarity, that is, $A \geq_{T} B \Leftrightarrow P^{T} A P \geq_{T} P^{T} B P$.

Result 2.1.9

Rank subtractivity ordering is preserved under unitary similarity, that is, $A \geq_{r s} B \Leftrightarrow P^{T} A P \geq_{r s} P^{T} B P$.

Theorem 2.1.10 similarity.

Lowener order, transpose order and rank subtractivity order are all preserved for s-k-orthogonal

Proof

(i) Lowener ordering is preserved for s-k-orthogonal similarity. We have to prove that, $A \geq_{L} B \Leftrightarrow K V P^{-1} K V A P \geq_{L} K V P^{1} K V B P$ for some orthogonal matrix $P$.

For $A \geq_{L} B \Leftrightarrow K V A \geq_{L} K V B$

(by Theorem (2.1.6))

$$
\begin{aligned}
& \Leftrightarrow P^{T} K V A P \geq_{L} P^{T} K V B P . \\
& \Leftrightarrow K V P^{T} K V A P \geq_{L} K V P^{T} K V B P . \\
& \Leftrightarrow\left(K V P^{-1} K V\right) A P \geq_{L}\left(K V P^{-1} K V\right) B P \\
& \Leftrightarrow C \geq_{L} D
\end{aligned}
$$

Where $C=K V P^{-1} K V A P$ is orthogonaly s-k-similar to $A$

$$
D=K V P^{-1} K V B P \text { is orthogonaly s-k-similar to } B
$$

Thus, Lowener ordering is preserved for s-k-orthogonal similarity.

(ii) Star ordering is preserved for s-k-orthogonal similarity, we have to prove that, $A \geq_{T} B \Leftrightarrow\left(K V P^{-1} K V\right) A P \geq_{T}\left(K V P^{-1} K V\right) B P$, for some orthogonal matrix $P$.

$$
\text { For } \begin{aligned}
A \geq_{T} B & \Leftrightarrow K V A \geq_{T} K V B \\
& \Leftrightarrow P^{T} K V A P \geq_{T} P^{T} K V B P \\
& \Leftrightarrow K V P^{T} K V A P \geq_{T} K V P^{T} K V B P \\
& \Leftrightarrow\left(K V P^{-1} V K\right) A P \geq_{T}\left(K V P^{-1} V K\right) B P .
\end{aligned}
$$

(by Theorem

(by result (2.1.8))

(by Theorem (2.1.6))

(iii) Rank subtractivity ordering is preserved for s-k-orthogonal similarity, we have to show that, $A \geq_{r s} B \Leftrightarrow\left(K V P^{-1} K V\right) A P \geq_{r s}\left(K V P^{-1} K V\right) B P$ for some orthogonal matrix $P$.

For, $A \geq_{r s} B \Leftrightarrow K V A \geq_{r s} K V B$

(by Theorem (2.1.6))

$$
\Leftrightarrow P^{T} K V A P \geq_{r s} P^{T} K V B P
$$

(by result (2.1.9)) 


$$
\begin{aligned}
& \Leftrightarrow K V P^{T} K V A P \geq_{r s} K V P^{T} K V B P \\
& \Leftrightarrow\left(K V P^{-1} K V\right) A P \geq_{r s}\left(K V P^{-1} K V\right) B P
\end{aligned}
$$

(by Theorem (2.1.6))

Thus rank subtractivity is preserved for s-k-orthogonal similarity. Thus all the three standard partial orderings are preserved for s-k-orthogonal similarity.

\section{References}

[1]. Baksalary, J.K., "Relationship between the star and minus orderings." Lin. Alg. Appl., 82 (1986), $163-168$.

[2]. Ben- Israel, A. and Greville, T.N.E., "Generalized Inverses: Theory and Applications." $2^{\text {nd }}$ Edition, Springer, New York (2003)

[3]. Hartwig, R.E. and Styan, G.P.H., "On some characterizations of the star partial ordering for matrices and rank subtractivity." Lin. Alg. Appl. 82 (1986), 145-161.

[4]. Hartwig. R.E. and Styan. G.P.H., "Partially ordered idempotent matrices." Proc. Second International Tampere Conference in Statistics, (1987), 361-383

[5]. Hauke, J. and Markiewicz, A., “On partial orderings on the set of rectangular matrices.” Lin. Alg. Appl., 219 (1995), $187-193$.

[6]. Krishnamoorthy, S., Gunasekaran, K. and Muthugobal, B.K.N., "Con-s-k-EP matrices", Journal of Mathematical Sciences and Engineering Applications, Vol. 5, No.1, 2011,353-364.

[7]. Mitra, S.K. and Puri, M.L., "The fundamental barded matrix of linear estimation and the Duffin Morley general linear electro mechanical systems.” Applicable Analys., 14 (1983), 241-258.

[8]. Mitra. S.K., "Matrix partial orderings through generalized inverses: unified theory.” Lin. Alg. Appl., 148 (1991), $237-263$.

[9]. Rao, C.R. and Mitra, S.K., “Generalized Inverse of Matrices and Its Applications”, Wiley and Sons, New York, 1971. 